\title{
Microcalcifications without a thyroid nodule as the sole sign of papillary thyroid carcinoma
}

\author{
${ }^{1}$ School of Medicine, University of Milan, Milan, Italy, ${ }^{2}$ Centre of Endocrine Surgery, Evangelistria Medical Centre, \\ Nicosia, Cyprus, ${ }^{3}$ University of Nicosia Medical School, Nicosia, Cyprus, ${ }^{4}$ Child Endocrine Care, Department of \\ Pediatrics, Aretaeio Hospital, Nicosia, Cyprus, 5epartment of Molecular Genetics, Function and Therapy, The Cyprus \\ Institute of Neurology and Genetics, Nicosia, Cyprus, 6 Histopathology and Cytology Medical Centre, Nicosia, Cyprus, \\ ${ }^{7}$ CEDM, Centre of Endocrinology, Diabetes and Metabolism, Limassol, Cyprus, ${ }^{8}$ Department of Diabetes, \\ Endocrinology \& Obesity Medicine, Salford Royal NHS Foundation \& University Teaching Trust, Salford, UK, and \\ ${ }^{9}$ Medical School, European University of Cyprus, Nicosia, Cyprus
}

Stamatina loakim 1 , Vasilis Constantinides 2,3 , Meropi Toumba ${ }^{4,5}$, Theodoros Lyssiotis ${ }^{6}$ and Angelos Kyriacou $7,8,9$

Correspondence

should be addressed

to A Kyriacou

Email

angelos5@doctors.org.uk

\section{Summary}

Our objective is to demonstrate the importance of considering microcalcifications even without evidence of nodules as a potential sign of malignancy. Current guidelines, such as those of the British Thyroid Association, acknowledge the clinical significance of microcalcifications only when found within nodules. In this case, they are considered a suspicious feature, classifying the nodules as U5 (i.e. high risk) where fine-needle aspiration biopsy (FNAB) is warranted, following the high likelihood of cancer in these nodules. In addition, there is a dearth of evidence of ultrasound scan (USS) detection of microcalcifications in the thyroid gland outside of nodules, along with their associated clinical implications. Yet, this clinical manifestation is not so infrequent considering that we do encounter patients in the clinic showing these findings upon ultrasound examination. Three patients who presented to our clinic with thyroid-related symptoms were shown to have areas of microcalcifications without a nodule upon sonographic evaluation of their thyroid gland. These incidentally detected hyperechoic foci were later confirmed to correspond to areas of papillary thyroid carcinoma (PTC) on histopathological examination of resected tissue following thyroidectomy. Four more cases were identified with sonographic evidence of microcalcifications without nodules and given their clinical and other sonographic characteristics were managed with active surveillance instead.

\section{Learning points:}

- Echogenic foci known as microcalcifications may be visible without apparent association to nodular structures.

- Microcalcifications without nodules may not be an infrequent finding.

- Microcalcifications are frequently indicative of malignancy within the thyroid gland even without a clearly delineated nodule.

- Empirically, the usual guidelines for the management of thyroid nodules can be applied to the management of microcalcifications not confined to a nodule, but such a finding per se should be classified as a 'high-risk' sign.

\section{Background}

Differentiated thyroid cancers account for over $90 \%$ of all thyroid malignancies and PTC is the most prevalent subtype. Thyroid sonography is considered the mainstay imaging technique in diagnosing thyroid cancer as it is readily accessible, inexpensive, and extremely sensitive in nodule detection.

Although thyroid nodules are a common incidental finding, malignancy is implicated only in $5-15 \%$ of 
them (1). Guidelines stratify the risk of malignancy based on various morphological characteristics visible on USS and nodule size, in order to guide clinical intervention $(1,2)$. Nevertheless, it is important to note that no specific feature is pathognomonic of the nature of the lesion. Ultimately, USS-guided fine needle aspiration may be deemed necessary for further cytological examination.

Sonographic detection of microcalcifications within thyroid nodules, even though not exclusive to, is considered highly suggestive of malignancy with specificity (85.8-95\%) and positive predictive value (41.8-94.2\%) (3). Particularly within a solid hypoechoic nodule or solid hypoechoic component of a partially cystic nodule, they carry an estimated risk of $70-90 \%$ for malignancy with recommendations for FNA at $\geq 1 \mathrm{~cm}$ (1). Notwithstanding, microcalcifications outside of nodular masses are not well documented. Microcalcifications on ultrasonography are depicted as punctate echogenic foci that lack posterior acoustic shadowing and measure up to $1 \mathrm{~mm}$ in diameter (3). They are believed to correspond to aggregates of psammoma bodies histologically, which are $10-100 \mu \mathrm{m}$, spherical and concentrically laminated calcifications. Even though the sole finding of psammoma bodies is not specific to PTC, in conjunction with a papillary architecture and specific nuclear modifications, they were found to have $100 \%$ positive predictive value (4). Despite their unclear pathogenesis, it has been proposed that they are involved in processes such as dystrophic calcification, intravascular tumor thrombi calcifications, malignant papillae infarction (5) and that they may hinder cancer growth (6).

Differentiation from other echogenic lesions is based on their sonographic features but limited by the spatial resolution of the image. Macrocalcifications such as coarse calcifications present with a posterior acoustic shadow whereas colloid is accompanied by the comet-tail artifact. Perception of these signs may be impaired by factors such as their depth within the tissue or the location of the transducer focal zone (5).

We hereby present three patients who presented with evident microcalcifications in the thyroid gland upon ultrasound examination, independent of nodule confinement. All three patients underwent thyroidectomy, with subsequent histopathological diagnosis of PTC corresponding to the areas of concern highlighted during the USS evaluation. Additionally, we briefly present four patients who also had microcalcifications without a nodule on sonography but who did not undergo any cytological nor histological examination.

\section{Case I presentation}

A 43-year-old male presented with mild conjunctival erythema. He was found to have subclinical hyperthyroidism and high levels of TSH receptor antibodies (TRAb). He was diagnosed with Graves' disease and mild thyroid eye disease.

\section{Investigation}

On initial ultrasound scan of the thyroid gland, normal thyroid appearance was noted, but neck examination revealed a suspicious right-sided level III lymph node measuring $6.3 \times 31.6 \mathrm{~mm}^{2}$ with microcalcifications, cystic components, and echogenic parenchyma (Fig. 1A). Repeat sonography in a joint clinic of two experienced consultants also revealed two spots of microcalcifications with no posterior acoustic shadowing in the right lobe of the thyroid (Fig. 1B).
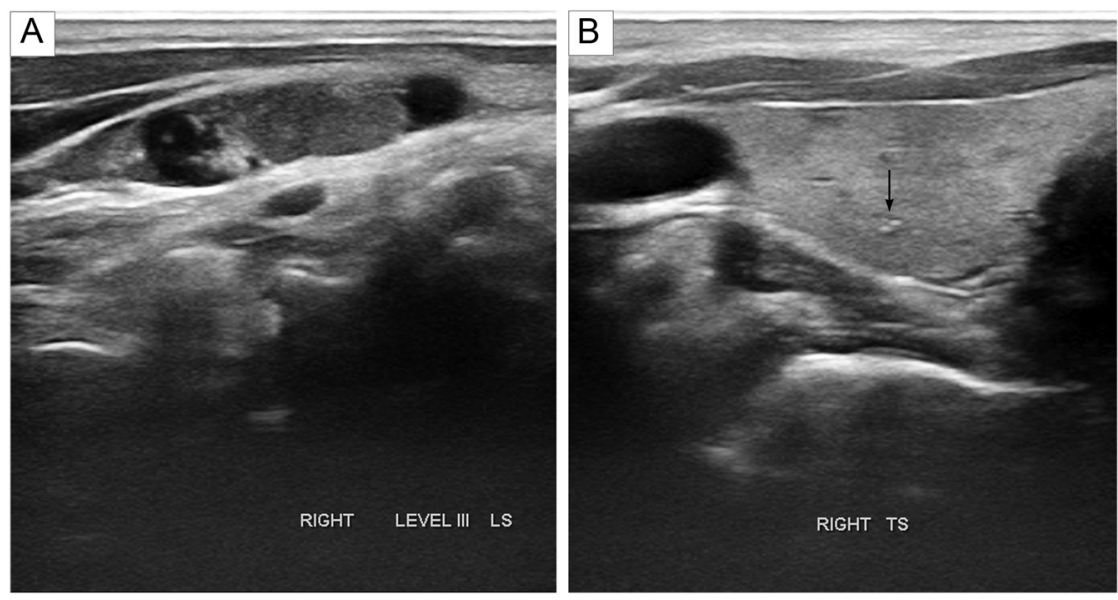

Figure 1

(A) Longitudinal view of a preoperative ultrasound image of level III lymph node with cystic components and increased echogenicity. (B) Transverse view of a preoperative ultrasound image of first patient showing microcalcifications depicted as punctate echogenic foci (arrow) without a nodule. Beware that ultrasound imaging provides real-time dynamic footage, whilst static images may give a misconception of surrounding heterogeneity; indeed, in this case, no nodule was observed on dynamic images. 


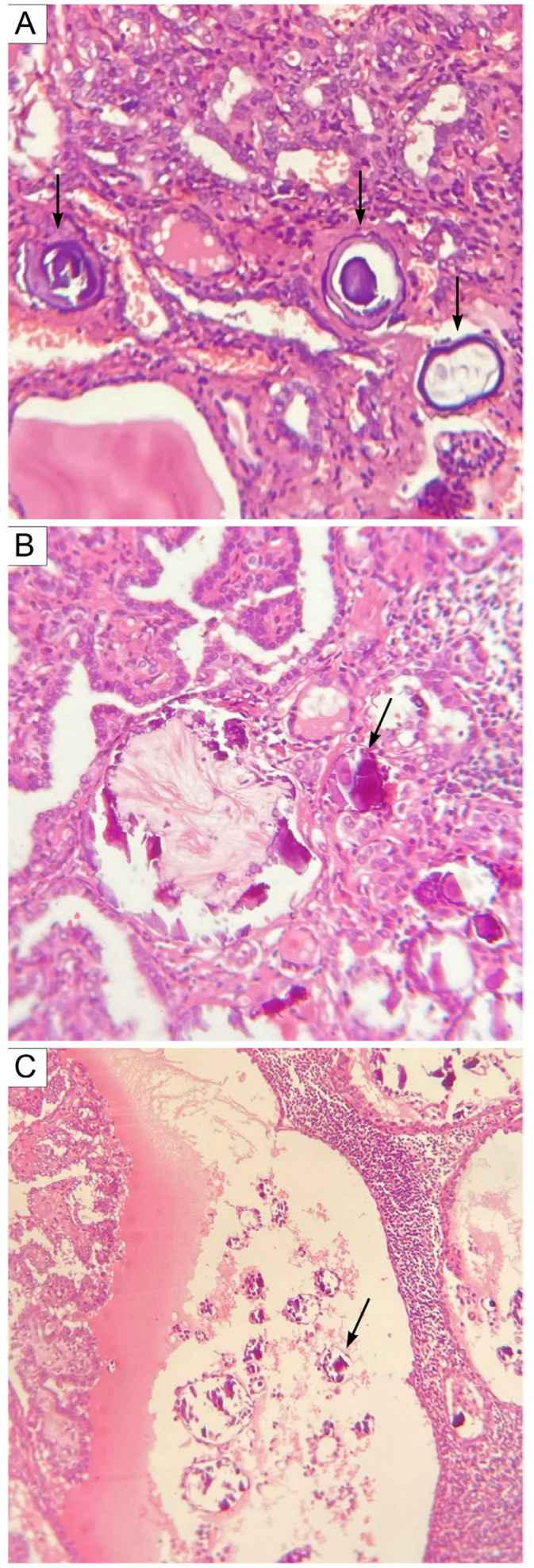

\section{Figure 2}

Histopathological analysis of tissue resected from the first patient with hematoxylin and eosin (H\&E) staining showing (A) psammoma bodies (arrows) entrapped between neoplastic papillary elements (H\&E, $\times 40)$, (B) calcifications of variable sizes (arrow) in sclerosing papillary thyroid carcinoma (H\&E, $\times 40)$, and (C) numerous psammoma bodies (arrow) scattered throughout cystic degeneration of a lymph node $(H \& E, \times 10)$.
FNAB of this lymph node was positive for PTC with cystic and hemorrhagic degeneration.

\section{Treatment}

The patient was referred for total thyroidectomy with right lateral and central compartment lymph node dissection. Histopathological examination revealed a right mid-lobe sclerosing type microPTC (3 $\mathrm{mm})$, showing papillary elements lined by abnormal cells and psammoma bodies (Fig. 2). The location of the carcinoma macroscopically and microscopically corresponded to the aforementioned sonographic microcalcifications. The tumor was peripheral, demonstrating microscopic extrathyroidal extension alongside central and lateral compartment lymph node positivity.

\section{Outcome and follow-up}

The TNM staging (AJCC 2016) for this tumor was pT3 N1b M0, stage I. Postoperative radioactive iodine (RAI) was administered and the patient has been well almost 3 years after surgery on an excellent response category as per the ATA guidelines.

\section{Case II presentation}

The second patient was a 28-year-old female presenting with neck swelling.

\section{Investigation}

A thyroid USS revealed one nodule in the right lobe as well as a poorly defined cluster of echogenic foci, consistent with microcalcifications, and without a visible surrounding nodule (Fig. 3A). Two right-sided level IV lymph nodes with suspicious features were detected during the USS neck exam.

Cytologic features of the two thyroid lesions categorized them as Thy 2 and Thy5 each.

\section{Treatment}

Following thyroidectomy, histology confirmed the suspected diagnosis of PTC and presence of psammoma bodies. The right lobe was distorted, enclosing two tumors, 8 and $4 \mathrm{~mm}$, respectively. Peri-thyroid tissue invasion from the larger tumor was noted while the smaller tumor showed infiltration of the thyroid capsule without extension into the peri-thyroid tissue. 

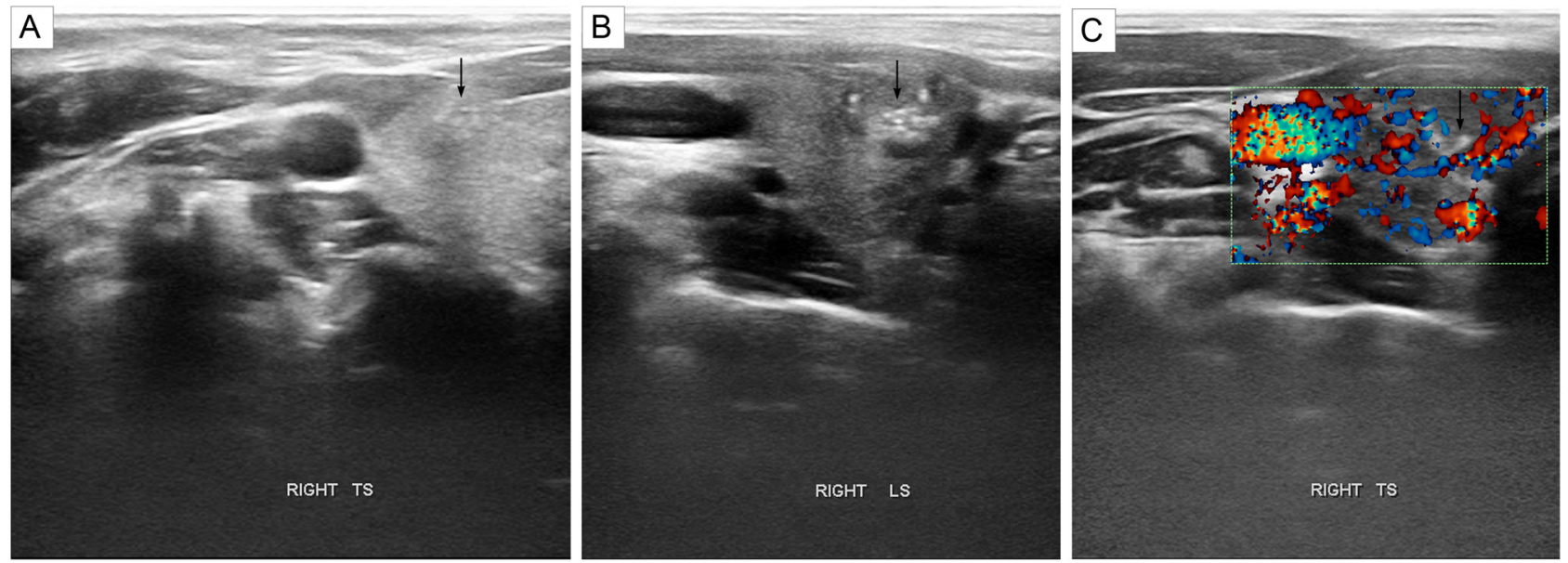

\section{Figure 3}

Preoperative ultrasound images of (A) second patient and (B) third patient with evident microcalcifications (arrow) in absence of associated nodules. Evidently increased vascularity in third patient's thyroid gland consistent with Graves' disease diagnosis (C), without any delineated structure of a nodule surrounding the echogenic foci (arrow).

\section{Outcome and follow-up}

This presentation was consistent with multifocal papillary carcinoma of the right thyroid lobe with 7 out of 17 lymph nodes on the right showing evidence of metastasis. TNM staging was pT3 N1b M0, stage I. Subsequently, RAI ablation was performed, and the patient remains well 1.5 years after surgery.

\section{Case III presentation}

The third patient was a 20-year-old female previously diagnosed with Graves' disease in a different center and who was receiving carbimazole.

\section{Investigation}

A routine neck USS revealed multiple increased echogenic foci suggestive of microcalcifications extending to the anterior capsule (Fig. 3B). No clear peripheral hypoechogenicity nor a surrounding halo was observed, and utilization of the Doppler function failed to reveal any well-delineated structure consistent with a nodule (Fig. 3C). The treating consultants were, therefore, confident to exclude the presence of a nodule since the cluster of microcalcifications was identified in the context of diffuse heterogenous hypoechogenicity. FNAB results indicated a Thy5 lesion of PTC.

\section{Treatment}

Total thyroidectomy with central compartment lymph node dissection was performed, histological examination of which confirmed the diagnosis of classical PTC.
Presence of diffuse psammoma bodies was reported along with macrocalcifications. The rest of the thyroid parenchyma showed the presence of sparse palpable granulomas and foci of lymphocytic thyroiditis.

\section{Outcome and follow-up}

Hence, TNM staging of the carcinoma was classified as pT1b N0 M0, stage I. The location of the microcalcifications on the USS coincided with the microscopic location and characteristics of PTC. The patient underwent RAI ablation and remains well 2 years after surgery.

At least four more patients who presented to our clinic in recent years were found to have extra-nodular microcalcifications on USS but we felt that there was no indication for an FNA examination of these nodules. Rather, we proceeded with active surveillance as per an agreed shared pathway with the patients. We, thereby, briefly present these patients.

A 34-year-old female presented because of a family history of metastatic thyroid cancer for which she received routine thyroid USS screening. Images revealed a cluster of microcalcifications without a nodule measuring 4.6 $\mathrm{mm} \times 3 \mathrm{~mm} \times 5 \mathrm{~mm}$, adjacent to another area of potential microcalcifications within a poorly defined hypoechoic area possibly corresponding to a nodule (Fig. 4A). Given the small size of the lesions and their intra-thyroidal location, FNA was not advised. She remains well with stable appearances of these lesions over 3 years of monitoring.

The next patient was a 43-year-old asymptomatic female who was also identified to have a small cluster of microcalcifications, in absence of a nodule, measuring 

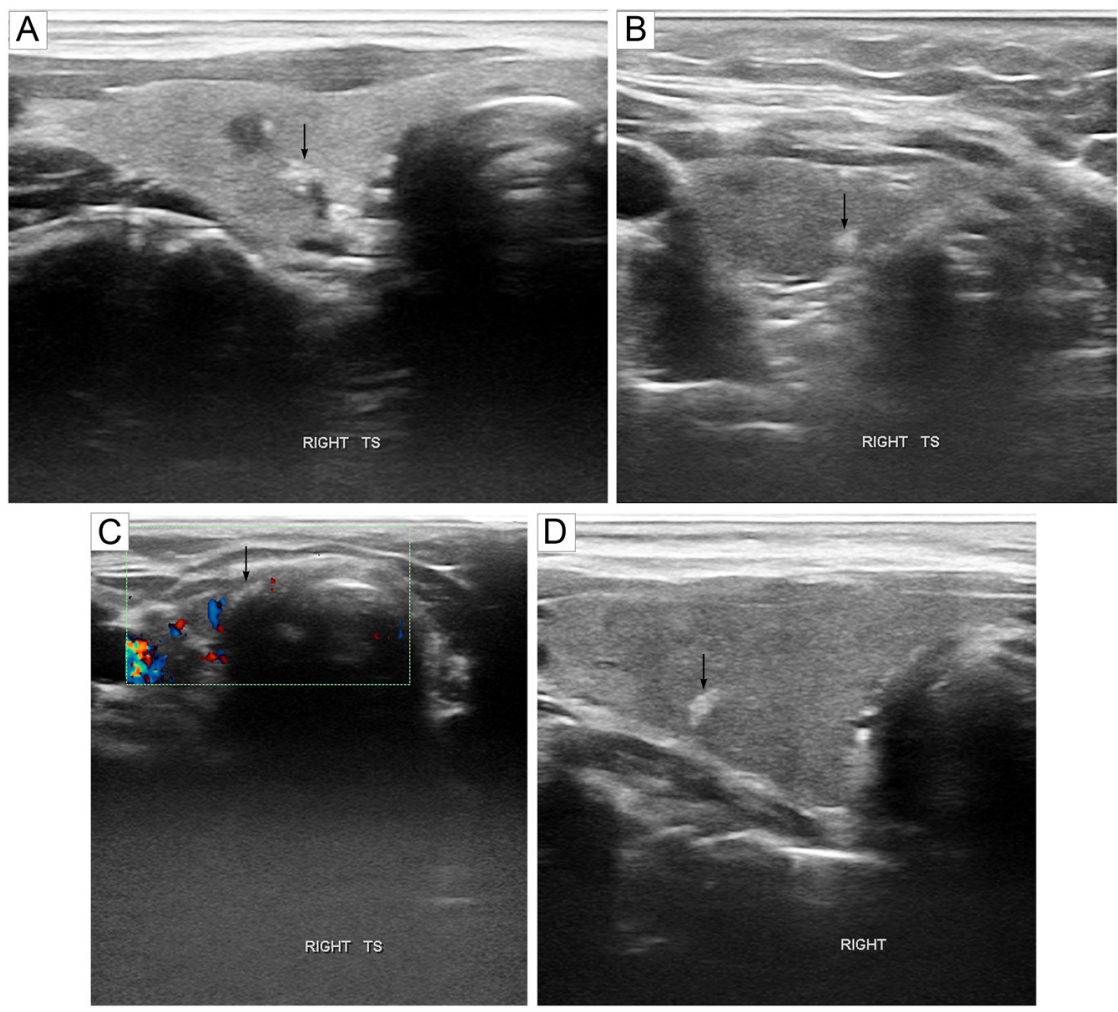

\section{Figure 4}

Ultrasound images of (A) fourth, (B) fifth, (C) sixth, and (D) seventh patient with small-sized clusters of microcalcifications (arrow) without nodules. The patients whose images are shown in $(A, B, D)$ showed small and intra-thyroidal collections of microcalcifications without nodules nor suspicious lymphadenopathy and as such there was no indication for FNA. Patient whose image is shown in (C) was offered FNA due to the close proximity of the lesion to the thyroid capsule, but she declined.

$3 \mathrm{~mm} \times 2.8 \mathrm{~mm}$ on transverse view (Fig. 4B). In view of the small size, intra-thyroidal location, and lack of any other concerning features, the treating physician advised to monitor the evolution of the lesion. The patient has since been lost to follow-up.

The sixth patient was a 69-year-old female who previously underwent partial thyroidectomy for a large multinodular goiter with benign appearances on histology. During a routine follow-up USS, microcalcifications were detected without a thyroid nodule, measuring $5.7 \mathrm{~mm} \times 3.3 \mathrm{~mm} \times 5.3 \mathrm{~mm}$ (Fig. 4C) FNA was suggested as the microcalcifications were in close contact with the capsule, but the patient declined. Sonographic surveillance showed no changes over 2 years of observation. Thereafter, the patient died of a primary brain tumor.

The other patient was a 63-year-old female which upon USS screening was found to have a cluster of microcalcifications measuring $4.7 \mathrm{~mm} \times 2.9 \mathrm{~mm} \times 2.7$ mm outside of a nodule (Fig. 4D). Since the lesion was small and intra-thyroidal, with the thyroid exhibiting an intact capsule and no lymphadenopathy, FNA was not recommended. Extrapolating from the current ATA guidelines (1), active surveillance was a valid option in this case. Patient remains well with stable appearances of her thyroid over 2 years of monitoring.

\section{Discussion}

The best evidence of the existence of this sonographic sign of microcalcifications comes from a South Korean study (7). In this retrospective study by Kwak et al., the sonographic images and/or notes of 2158 patients with thyroid carcinoma were reviewed. The authors identified 11 cases where the sole suspicious USS observation was microcalcifications in the absence of an associated mass. Following thyroidectomy, seven of these cases were diagnosed with classical PTC whereas the other four cases presented with the diffuse sclerosing variant of PTC. Cervical lymph node metastases were detected in $43 \%$ of patients with classical PTC compared to $75 \%$ of patients with diffuse sclerosing PTC implying a higher incidence of metastasis in the latter variant. Thus, in a cohort of 2158 thyroid cancer patients, $0.5 \%$ of them had no other alarming sign for malignancy aside from isolated microcalcifications.

A Chinese study (8) investigated the distribution of microcalcifications identified on ultrasound in 358 cases of suspected thyroid cancer. Records of previously performed scans were contrasted to the histopathological specimens in order to identify patterns indicative of malignancy. Thirty-one cases involving microcalcifications alone were identified, 12 of which had a focal distribution while a 
diffuse distribution was noted for the remaining 19. The authors reported that 7/12 cases with focal distribution were diagnosed with PTC after a pathological examination. The other five cases were found to have benign Hashimoto thyroiditis. Conversely, diffuse microcalcifications were reported to have $100 \%$ specificity for malignancy since all those cases had PTC.

A recently published retrospective study (9) investigated a database of 2698 FNABs and identified 21 patients with USS evidence of clustered or scattered microcalcifications in the absence of associated thyroid nodules, who underwent thyroidectomy. Cancer was diagnosed in $62 \%$ of these patients. Almost all cases were attributed to classical PTC except one case of tallcell variant PTC. The authors reported that $2 \%$ of all PTC diagnoses were accompanied by this particular sonographic finding, which bearing in mind the high prevalence of PTC, would be a relevant finding in a considerable number of patients.

Overall, the research conducted on this phenomenon is limited. Consequently, currently published guidelines on the management of thyroid malignancies only address the significance of microcalcifications when observed within nodules or lymph nodes. However, our cases alongside the aforementioned studies demonstrate the clinical implications of this particular sign. Further research is indicated to describe the exact prevalence and significance of this phenomenon. In the first three patients reported above, the spatial distribution of the microcalcifications detected incidentally during US imaging of the thyroid was consistent with the areas of PTC described during histology. The findings in our patients emphasize the validity of this peculiar sonographic pattern. Imaging detection of malignancy in the thyroid is predominantly performed through assessing the morphology of nodules. Nonetheless, attention should be drawn to suspicious observations even in the absence of nodules as in the cases mentioned above. Identification of psammoma bodies seems to coincide with increased PTC aggressiveness (10). Association between psammoma bodies in the setting of PTC and nodal involvement has been reported, as observed in our first two patients.

Worth noting is the fact that two out of our three patients with PTC diagnosis had underlying Graves' disease. When found in concomitance with thyroid nodules, Graves' disease likely confers an increased risk for thyroid carcinoma (11). Therefore, the implementation of routine USS in all patients with Graves' disease has been proposed (12). It could be argued that this practice would incur extra costs or subject patients to unnecessary medical interventions but as in the aforementioned cases, it could lead to the early detection of signs of malignancy. A systematic review and meta-analysis of seven retrospective studies involving 2528 patients with Graves' disease demonstrated that $11.5 \%$ (297) had thyroid cancer (11). Screening can, therefore, benefit a substantial proportion of Graves' disease patients with an early cancer diagnosis which may impact the management strategy of hyperthyroidism.

In summary, we report the cases of seven patients with sonographic evidence of hyperechoic punctate lesions within the thyroid gland without association to nodules. The pathohistological examination that was performed in the first three cases verified the presence of PTC and its association to the previously observed microcalcifications. No pathological diagnosis is available for the remaining patients since active surveillance was considered to be the most appropriate clinical action. The above cases demonstrate that the presence of microcalcifications without a nodule is not an infrequent finding on sonography. Current guidelines do not address these hyperechoic lesions in absence of nodules but nonetheless, they should be viewed as 'high-risk' features.

\section{Declaration of interest}

The authors declare that there is no conflict of interest that could be perceived as prejudicing the impartiality of this case report.

\section{Funding}

This work did not receive any specific grant from any funding agency in the public, commercial or not-for-profit sector.

\section{Patient consent}

Written consent has been obtained from all patients.

\section{Author contribution statement}

A K conceived the idea and supervised the writing. S I wrote the first draft. A $\mathrm{K}, \mathrm{M} \mathrm{T}$ and $\mathrm{V} C$ had input in the care of the involved patients. $\mathrm{T} L$ performed the histopathological analysis of the specimens. All authors have viewed and contributed to the final manuscript.

\section{References}

1 Haugen BR, Alexander EK, Bible KC, Doherty GM, Mandel SJ, Nikiforov YE, Pacini F, Randolph GW, Sawka AM, Schlumberger M, et al. American Thyroid Association management guidelines for adult patients with thyroid nodules and differentiated thyroid cancer: the American Thyroid Association guidelines task force on thyroid nodules and differentiated thyroid cancer. Thyroid 201626 1-133. (https://doi.org/10.1089/thy.2015.0020) 
2 Perros P, Boelaert K, Colley S, Evans C, Evans RM, Gerrard Ba G, Gilbert J, Harrison B, Johnson SJ, Giles TE, et al. Guidelines for the management of thyroid cancer. Clinical Endocrinology $2014 \mathbf{8 1}$ (Supplement 1) 1-122. (https://doi.org/10.1111/cen.12515)

3 Hoang JK, Lee WK, Lee M, Johnson D \& Farrell S. US features of thyroid malignancy: pearls and pitfalls. RadioGraphics 200727 847-861. (https://doi.org/10.1148/rg.273065038)

4 Pusztaszeri MP, Auger M, Stelow EB, Yang GCH, Sanchez MA \& LiVolsi VA. Papillary thyroid carcinoma, variants, and related tumors. In The Bethesda System for Reporting Thyroid Cytopathology, pp. 119-156. Springer International Publishing, 2018. (https://doi.org/10.1007/9783-319-60570-8_8)

5 Tahvildari AM, Pan L, Kong CS \& Desser T. Sonographic-pathologic correlation for punctate echogenic reflectors in papillary thyroid carcinoma: what are they? Journal of Ultrasound in Medicine 201635 1645-1652. (https://doi.org/10.7863/ultra.15.09048)

6 Das DK. Psammoma body: a product of dystrophic calcification or of a biologically active process that aims at limiting the growth and spread of tumor? Diagnostic Cytopathology 200937 534-541. (https://doi. $\operatorname{org} / 10.1002 /$ dc.21081)

7 Kwak JY, Kim EK, Son EJ, Kim MJ, Oh KK, Kim JY \& Kim KI. Papillary thyroid carcinoma manifested solely as microcalcifications on sonography. American Journal of Roentgenology 2007189 227-231. (https://doi.org/10.2214/AJR.06.0750)

8 Ning CP, Ji QL, Fang SB, Wang HQ, Zhong YM \& Niu HT. Distribution patterns of microcalcifications in suspected thyroid carcinoma: a classification method helpful for diagnosis. European Radiology 2018 28 2612-2619. (https://doi.org/10.1007/s00330-017-5212-2)

9 Whittle C, García M, Horvath E, Slater J \& Carrasco C. Thyroid microcalcifications in the absence of identifiable nodules and their association with thyroid cancer. Journal of Ultrasound in Medicine 2019 38 97-102. (https://doi.org/10.1002/jum.14667)

10 Pyo JS, Kang G, Kim DH, Park C, Kim JH \& Sohn JH. The prognostic relevance of psammoma bodies and ultrasonographic intratumoral calcifications in papillary thyroid carcinoma. World Journal of Surgery 201337 2330-2335. (https://doi.org/10.1007/s00268-013-2107-5)

11 Papanastasiou A, Sapalidis K, Goulis DG, Michalopoulos N, Mareti E, Mantalovas S \& Kesisoglou I. Thyroid nodules as a risk factor for thyroid cancer in patients with Graves' disease: a systematic review and metaanalysis of observational studies in surgically treated patients. Clinical Endocrinology 201991 571-577. (https://doi.org/10.1111/cen.14069)

12 Kyriacou A, Syed AA \& Economides PA. Thyroid sonography as an extension of the bedside examination in hyperthyroidism. Journal of Medical Science 202089 e482. (https://doi.org/10.20883/medical.e482)

Received in final form 3 August 202

Accepted 19 August 2021 